\title{
The ratio of serum Angiopoietin-1 to Angiopoietin-2 in patients with cervical cancer is a valuable diagnostic and prognostic biomarker
}

\footnotetext{
Ping Yang ${ }^{1,2}$, Na Chen ${ }^{1}$, Dongyun Yang ${ }^{1}$, Janet Crane ${ }^{3,4}$, Shouhua Yang ${ }^{1}$, Hangyu Wang ${ }^{5}$, Ruiqing Dong , Xiaoqing Yi ${ }^{1}$, Lisha Xie ${ }^{1}$, Guo Jing ${ }^{1}$, Jing Cai ${ }^{1}$, Zehua Wang ${ }^{\text {Corresp. }}{ }^{1}$

1 Department of Obstetrics and Gynecology, Union Hospital, Tongji Medical College, Huazhong University of Science and Technology, Wuhan, China

2 Department of Obstetrics and Gynecology, First Affiliated Hospital, School of Medicine, Shihezi University, Shihezi, China

3 Department of Orthopaedic Surgery,, Johns Hopkins University School of Medicine, Baltimore, MD, USA

4 Department of Pediatrics, Johns Hopkins University, Baltimore, MD, USA

5 School of Pharmacy, Shihezi University, Shihezi, Xinjiang, China

6 Department of Obstetrics and Gynecology, Tianyou Hospital attended to Wuhan University of Science and Technology, Wuhan, Hubei, China

Objectives: Angiopoietins have found to play essential roles in tumor angiogenesis. The present study was aimed to investigate the diagnostic and prognostic values of serum angiopoietin 1 and 2 (sAng-1 and sAng-2) in cervical cancer. Methods: The sAng-1 and sAng-2 concentrations were analyzed in 77 women with cervical cancer, 44 women with cervical intraepithelial neoplasia (CIN) and 43 women without cervical lesions by enzymelinked immunosorbent assay. The diagnostic values of sAng-1, sAng-2 and sAng-1/sAng-2 were evaluated by receiver operating characteristic (ROC) curves. The Ang-1 and Ang-2 expression, as well as microvessel density (MVD), in cervical cancer tissues were assessed by immunohistochemistry. Results: The concentration of sAng-2 gradually increased and the sAng-1/Ang-2 ratio was gradually decreased from normal control to $\mathrm{CIN}$, then to squamous cell cancer, and the sAng-1/sAng-2 ratio was also significantly decreased in adenocarcinoma. The area under ROC curves of sAng- 2 and sAng-1/sAng- 2 ratio for discriminating cervical cancer from normal were 0.744 and 0.705 , respectively. Decreased sAng-1/sAng-2 was significantly associated with advanced tumor stage, poor differentiation, lymph-vascular space invasion and high MVD. sAng-2 was positively correlated with the Ang-2 expression in cervix epithelia. A high sAng-1/sAng-2 ratio was associated with a longer progression-free survival and a longer overall survival in cervical cancer patients. Conclusions: These findings suggest that sAng- 2 and the sAng-1/sAng- 2 ratio may be valuable diagnostic and prognostic biomarkers for cervical cancer. 
1 The ratio of serum Angiopoietin-1 to Angiopoietin-2 in patients with cervical cancer is a

2 valuable diagnostic and prognostic biomarker

4 Ping Yang ${ }^{1,2}$, Na Chen ${ }^{1}$, Dongyun Yang ${ }^{1}$, Janet Crane ${ }^{3,4}$, Shouhua Yang ${ }^{1}$, Hangyu Wang 5 ,

5 Ruiqing Dong ${ }^{6}$, Xiaoqing $\mathrm{Yi}^{1}$, Lisha Xie ${ }^{1}$, Guo Jing ${ }^{1}$, Jing Cai ${ }^{1}$, Zehua Wang ${ }^{1 *}$

1. Department of Obstetrics and Gynecology, Union Hospital, Tongji Medical College, Huazhong University of Science and Technology, Wuhan 430022, PR China.

2. Department of Obstetrics and Gynecology, First Affiliated Hospital, School of Medicine, Shihezi University, Shihezi 832008, PR China.

3. Department of Orthopaedic Surgery, Johns Hopkins University School of Medicine, Baltimore, MD, USA

4. Department of Pediatrics, Johns Hopkins University, Baltimore, MD, USA

5. School of Pharmacy, Shihezi University, Shihezi 832002, PR China

6. Department of Obstetrics and Gynecology, Tianyou Hospital attended to Wuhan University of Science and Technology, Wuhan 430022, PR China

*Correspondence to: Zehua Wang, Department of Obstetrics and Gynecology, Union Hospital, Tongji Medical College, Huazhong University of Science and Technology, 1277 Jiefang Avenue, Wuhan 430022, PR China. E-mail: zehuawang@,163.net

\section{$\underline{\text { Abbreviation }}$}

angiopoietin, Ang; serum Ang-2, sAng-2; vascular endothelial growth factor, VEGF; cervical intraepithelial neoplasia, CIN; microvessel density, MVD; cervical squamous cell carcinoma, CSCC; cervical adenocarcinoma, CADC; lymphovascular space invasion, LVSI; Progressionfree survival, PFS; Overall survival, OS; enzyme-linked immunosorbent assay, ELISA; Horseradish Peroxidase, HRP; immunohistochemistry, IHC; diaminobenzidine, DAB; 
endothelial cells, EC; high power field, HP.

\section{Abstract}

Objectives: Angiopoietins have found to play essential roles in tumor angiogenesis. The present study was aimed to investigate the diagnostic and prognostic values of serum angiopoietin 1 and 2 (sAng-1 and sAng-2) in cervical cancer. Methods: The sAng-1 and sAng-2 concentrations were analyzed in 77 women with cervical cancer, 44 women with cervical intraepithelial neoplasia (CIN) and 43 women without cervical lesions by enzyme-linked immunosorbent assay. The diagnostic values of sAng-1, sAng-2 and sAng-1/sAng-2 were evaluated by ROC. In addition, the Ang-1 and Ang-2 expression, as well as microvessel density (MVD), in cervical cancer tissues were assessed by immunohistochemistry. Results: The concentration of sAng-2 gradually increased and the sAng-1/sAng-2 ratio was gradually decreased from normal control to CIN, then to squamous cell cancer, and the sAng-1/sAng-2 ratio was also significantly decreased in adenocarcinoma. The area under ROC curves of sAng-2 and sAng-1/sAng-2 ratio for discriminating cervical cancer from normal were 0.744 and 0.705 , respectively. Decreased sAng1/sAng-2 was significantly associated with advanced tumor stage, poor differentiation, lymphvascular space invasion and high MVD. sAng-2 was positively correlated with the Ang-2 expression in cervix epithelia. A high sAng-1/sAng-2 ratio was associated with a longer progression-free survival and a longer overall survival in cervical cancer patients. Conclusions: These findings suggest that sAng-2 and the sAng-1/sAng-2 ratio may be valuable diagnostic and prognostic biomarkers for cervical cancer.

\section{Introduction}

Globally, cervical cancer incidence has increased from 378,000 cases per year in 1980 to 528,000 cases per year in 2012, representing a $0.6 \%$ annual rate of increase (Ferlay et al. 2015). The majority of cervical cancer patients come from the economically underdeveloped areas, and 
they have higher mortality. Although the cervical screening programs based on the cervical cytology and HPV test can effectively reduce the incidence of cervical cancer, women in underdeveloped areas usually cannot accept standardized screening due to social, religious and psychological factors. Thus, novel circulating biomarkers that allow monitoring of essential molecular events in cervical cancer may improve the detection of lesions that have a high risk of progression in both primary screening and triage settings.

Angiogenesis is essential for tumor progression and has shown great promise as a therapeutic target for treatment of advanced cervical cancer (Eklund \& Saharinen 2013; Kerbel 2015). Recently, agents targeting the angiopoietin (Ang)/Tie system have been developed in several clinical trials and achieved encouraging results in anti-angiogenesis therapy (Gerald et al. 2013). The human Ang/Tie system is mainly composed of three secreted ligands (Ang-1, Ang-2, and Ang-4) and two tyrosine kinase receptors Tie-1 and -2. All three human angiopoietins bind directly to Tie-2 and result in phosphorylation and multimerization of Tie-2, which in turn trigger downstream signaling and regulate vascular remodeling and maturation. Ang-1 functions as a constitutive Tie-2 receptor agonist. The function of Ang-2 is context-dependent; Ang-2 functions as an partial Tie-2 signaling agonist in the presence of Ang-1 or a Tie-2 signaling agonist in the absence of Ang-1 (Augustin et al. 2009; Fagiani \& Christofori 2013; Yang et al. 2015). Ang-1 is widely expressed in periendothelial cells in human quiescent vasculature, where it sustains vessel maturation and stabilization. In contrast, Ang-2 is expressed in endothelial cells during vascular remodeling and angiogenesis, where it mediates vascular destabilization. Thus, the balance of Ang-1 and Ang-2, as well as the cellular context of Tie-2 activation, contribute to the outcome of Tie-2 activity. In addition, angiopoietins have been shown to directly interact with integrins on endothelial, mesenchymal and tumor cells, thereby eliciting Tie-2-independent biological effects (Augustin et al. 2009; Fagiani \& Christofori 2013; Shim et al. 2007). Both Ang-1 and -2 have recently been found to be expressed in tumor cells, and they act cooperatively with VEGF promoting the angiogenesis during tumor progression (Augustin et al. 2009; Ebos \& Kerbel 2011; 
82

83

Gerald et al. 2013; Shim et al. 2007; Yang et al. 2015).

Increased circulating serum Ang-2 (sAng-2) concentrations or a shift in the sAng-1/sAng-2 ratio in favor of sAng-2 in tumor patients has been shown to correlate with advanced tumor progression and poor survival in epithelial ovarian cancer (Sallinen et al. 2014; Sallinen et al. 2010), melanoma (Helfrich et al. 2009), metastatic colorectal cancer (Goede et al. 2010), neuroendocrine tumors (Detjen et al. 2010), hepatocellular carcinoma (Bouattour et al. 2015) and pancreatic cancer (Schulz et al. 2011). Furthermore, a soluble Tie-2 receptor can sequester angiopoietins (Augustin et al. 2009; Schulz et al. 2011). In cervical cancer, based on a preliminary study on plasma concentrations of Ang-land Ang-2 in 34 patients with cervical cancer, circulating Ang-1, Ang-2, and Tie-2 and the Ang-1/Ang-2 ratio were significantly increased compared with healthy women (Kopczynska et al. 2009). Moreover, decreased Ang1/Ang-2 ratio was associated with advanced Federation of Gynecology and Obstetrics (FIGO) stage (Kopczynska et al. 2009). However, the diagnostic and predictive value of circulating Ang1 and Ang-2 in cervical cancer remains largely unclear.

In the present study, the diagnosis and prognostic value of sAng- $1,-2$ and sAng- $1 / \mathrm{sAng}-2$ in patients with cervical cancer was evaluated. In addition, the correlations between the circulating angiopoietin concentrations and the angiopoietin expression levels and microvessel density (MVD) in tumor were investigated.

\section{Materials and methods}

\subsection{Study groups and inclusion criteria}

We conducted a non-matched case-control study using serum samples obtained from patients who received treatment at Wuhan Union Hospital (Wuhan, Hubei, China) between February 2012 to March 2014, including 44 patients with CIN, 77 patients with cervical cancer (61 
108

109

110

111

112

113

114

115

116

117

118

119

120

121

122

123

124

125

126

127

128

129

130

131

132

133

134

squamous cell carcinomas, CSCC, and 16 adenocarcinoma, CADC) and 43 patients with benign disorders of uterus such as myoma or adenomyosis as controls. The diagnoses were pathologically verified and patients with medical diseases such as other type of cancer or inflammatory, atherosclerotic and connective tissue disease were excluded. The age range of the 164 patients was 23-72 years with mean of 44.26 years, and there was no significant difference in age among the cervical cancer, CIN and control groups. All patients or their next of kin provided written informed consent for the collection of samples and subsequent research. The present study was approved by the Ethics Committee of Tongji Medical College, Huazhong University of Science and Technology (IORG0003571).

\subsection{Clinical definitions}

The medical records of the patients with cervical cancer were reviewed to collect clinical and pathological characters, including age at diagnosis, FIGO stage, pathological type, tumor differentiation, pelvic lymph node metastasis, tumor size, and lymphovascular space invasion (LVSI). The patients were classified according to the revised FIGO staging system for cervical cancer of 2009. The tumor size was the largest diameter of tumor determined by the attending gynecologic oncologist during a pelvic examination preceding surgery. The patients of IA1 stage underwent hysterectomy, and patients between IB1 and IIB underwent radical hysterectomy and pelvic lymph node dissection. Patients with $\geq$ IIB stage underwent radiotherapy or radiotherapy combined with chemotherapy. Stage-specific follow-up after treatment was performed in accordance with the FIGO guidelines. Progression-free survival (PFS) was defined as the time from treatment to the first appearance of tumor recurrence or to the date of last contact. Overall survival (OS) was defined as the time from treatment to death from any cause or to the date of last contact.

\subsection{Sample collection, ELISA, and IHC}

Serum samples were collected before primary treatment such as surgery, radiation, or 
135

136

137

138

139

140

141

142

143

144

145

146

147

148

149

150

151

152

153

154

155

156

157

158

159

160

161

chemotherapy. Blood was drawn into serum tubes $(10 \mathrm{ml})$ and centrifuged at $2200 \mathrm{G} / \mathrm{min}$ for 10 minutes. Serum was harvested, aliquoted and stored at $-80^{\circ} \mathrm{C}$ until usage.

The concentration of sAng-1 and sAng- 2 in serum was assayed by a standardized sandwich enzyme-linked immunosorbent assay (ELISA) in triplicate according to the protocol recommended by the manufacturer (Uscn Life Science Inc. Wuhan, China).

For immunohistochemistry (IHC), paraffin-embedded sections $(4 \mu \mathrm{m})$ were hydrated by alcohol and then subjected to antigen retrieval in $10 \mathrm{mM}$ sodium citrate buffer ( $\mathrm{pH}$ 6.0). After being treated with $3 \% \mathrm{H}_{2} \mathrm{O}_{2}$ for 10 minutes, samples were incubated with primary antibodies overnight at $4{ }^{\circ} \mathrm{C}$. Three primary antibodies were used: human antibodies for detection of Ang-1(AF923, Polyclonal, Goat IgG), Ang-2 (AF623, Polyclonal, Goat IgG) and CD34 (AF7227, Polyclonal, Sheep IgG) in cervical tissue were all purchased from R\&D (Minneapolis, MN, USA); the working concentrations of antibodies were $5 \mu \mathrm{g} / \mathrm{ml}$. Standard horseradish peroxidase staining using an appropriate biotinylated secondary antibody (ZSGB-BIO, Peking, PRC), the elite ABC kit (Avidin: Biotinylated enzyme Complex; ZSGB-BIO, Peking, PRC) and diaminobenzidine (ZSGB-BIO, Peking, PRC) were performed according to the manufacturers' instructions. Sections were counterstained with Gill's haematoxylin (Sigma, Aldrich, St. Louis, MO USA). The expression of angiopoietins in epithelial cells and endothelial cells (EC) in cervical tissues were separately evaluated and scored as negative staining and positive staining. MVD was assessed using CD34 (Yang et al. 2009).

\subsection{Statistical analysis}

Statistical analysis was carried out with SPSS 13.0 statistical software (SPSS, Chicago, IL, USA). Continuous variables were expressed as median and range; the data from each category was presented as frequency and percentage. Non-parametric tests were used for comparisons between different groups. The differences in sAng-1, sAng-2, and sAng-1/sAng-2 ratio between groups 
162

163

164

165

166

167

168

169

170

171

172

173

174

175

176

177

178

179

180

181

182

183

184

185

186

187

188

were analyzed with the Kruskal-Wallis test combined with Dunn's multiple comparison, the Jonckheere-Terpstra test or Mann-Whitney U test, as appropriate. ROC curve was used to analyze the diagnostic value of individual factors. Correlations were analyzed with the Spearman's correlation coefficient. Survival analyses were performed and Kaplan-Meier survival curves were generated; the survival distributions were compared by log-rank test. All tests were two-tailed, and a $P$ value of less than 0.05 was considered to be statistically significant.

\section{RESULTS}

\section{1 sAng-2 concentration and sAng-1/sAng-2 ratio are altered in patients with cervical} neoplasia

The sAng-1and sAng-2 in 43 patients of normal control, 44 CIN patients and 77 cervical cancer patients (61 CSCC patients and 16 CADC patients) were detected by ELISA. Given that CINs are the precancerous lesions of CSCCs, we first compared the sAng-1, sAng-2 and sAng-1/sAng2 ratio in the normal, CIN and CSCC patients. We found that the sAng-1 concentration was not significantly different between these three groups, with a median concentration of $58.13 \mathrm{pg} / \mathrm{ml}$ (range, 6.79-194.1 pg/ml) in the control group, $28.53 \mathrm{pg} / \mathrm{ml}$ (range, 7.22-248.5 pg/ml) in the CIN group and $37.79 \mathrm{pg} / \mathrm{ml}$ (range, 1.57-361.2 pg/ml) in the CSCC group (Kruskal-Wallis test, $P=0.068$, Fig. 1A). However, there was a significant difference in sAng-2 between the three groups (Kruskal-Wallis test, $P=0.000)$, and from normal control $(28.87(1.86-235.7) \mathrm{pg} / \mathrm{ml})$, to CIN (44.35(0.70-395.6) $\mathrm{pg} / \mathrm{ml})$, then to CSCC (109.90(1.09-431.70) $\mathrm{pg} / \mathrm{ml})$, in that with the increasing severity of cervical lesions, the concentration of sAng-2 gradually increased (Jonckheere-Terpstra test, $P<0.001$; Fig. 1B). The sAng-1/sAng-2 ratio was also significantly different between the three groups (Kruskal-Wallis test, $P<0.001$; Fig. $1 \mathrm{C}$ ). The sAng-1/sAng-2 ratio decreased gradually from the control group (median, 2.07; range, $0.25-30.36$ ), to the CIN group (median, 0.85; range, 0.08-17.54), then to the CSCC group (median,0.54; range, 0.0242.91) (Jonckheere-Terpstra test, $P<0.001$; Fig. 1C). 
We then compared the concentration of sAng-1and sAng-2 and the sAng-1/sAng-2 ratio in the CADC group relative to the normal groups. The differences in sAng-1(37.79 vs $58.13 \mathrm{pg} / \mathrm{ml})$ and sAng-2 concentrations (62.97 vs $28.87 \mathrm{pg} / \mathrm{m}$ ) were not significant (Fig. 1A, B). However, the sAng-1/sAng-2 ratio was significantly decreased in CADC patients compared with the normal group (0.58(0.06-33.33) vs 2.07(0.25-30.36), $P=0.008)$ (Fig. 1C).

\section{2 sAng-2 concentration and the sAng-1/sAng-2 ratio are valuable diagnostic biomarkers} for cervical lesions

Having determined the differential sAng-2 concentrations and sAng-1/sAng-2 ratio in cervical cancer lesions compared with normal, we then used ROC curves to test whether the sAng-2 concentration and sAng-1/sAng-2 ratio can serve as biomarkers to distinguish CSCC from normal and CIN. The data of the 43 patients of normal control, the 44 CIN patients, and the 61 CSCC patients were included in the analysis. The area under curve (AUC) of the ROC curves of sAng-2 for discriminating CSCC from normal (Fig. 2A), CSCC from CIN (Fig. 2B), CSCC from normal and CIN (Fig. 2C), CIN and CSCC from normal (Fig. 2D), and invasive cervical cancer from normal (Fig. 2E) were 0.769, 0.662, 0.715, 0.706 and 0.744, respectively. The sAng-2 cutoff value of $48.24 \mathrm{pg} / \mathrm{ml}$ provides a high diagnostic accuracy to discriminate CSCC from normal, with a sensitivity of $76.67 \%$ and a specificity of $74.42 \%$ (Fig. 2 F).

The AUC value of sAng-1/ sAng-2 as a diagnostic biomarker for normal to CIN, normal to CSCC, normal to CIN and CSCC and normal to cervical cancer was 0.657, 0.719, 0.693 and 0.705 (Fig. 2G-J), respectively. The cutoff value of 1.09 had a sensitivity of $68.83 \%$ and specificity of $72.09 \%$ to distinguish CSCC from normal (Fig. 2K).

\section{3 sAng-1 and sAng-2 correlate to clinicopathological characteristics in patients with} cervical cancer

The correlations between the concentrations of serum angiopoietins and clinicopathological 
216

217

218

219

220

221

222

223

224

225

226

227

228

229

230

231

232

233

234

235

236

237

238

239

240

241

242

characteristics of cervical cancer were analyzed in a subset of 77 patients with cervical cancer, including 41 stage IA1 to IB1, 20 stage IB2 to IIA and 16 stage $\geq$ IIB based on classification criteria (FIGO, 2009). Characteristics of the patients are summarized in Table 1. We found that sAng-1 concentration was significantly decreased in groups of stage $\geq \mathrm{IB} 2$, tumor size $>2 \mathrm{~cm}$ and LVSI compared with stages IA1-IB1, tumor size $\leq 2 \mathrm{~cm}$, or no LVSI, respectively. sAng-1 concentrations had no significant correlation with differentiation and pelvic lymphatic status. Although the sAng-2 concentration was significantly increased in the poorly differentiated group, no significant correlation with other clinicopathological characteristics were noted. sAng$1 /$ sAng-2 was significantly decreased in stages $\geq \mathrm{IB} 2$, poor differentiation and LVSI, but had no significant correlation with pelvic lymphatic metastasis status and tumor size (Table 2).

\section{4 sAng-2 concentration positively correlates with Ang-2 expression on epithelia and MVD in cervical tissues}

In a subset of 25 patients with invasive cervical cancer (FIGO stage IA2-IIA) and 10 normal control patients, angiopoietin expression and MVD were evaluated in paraffin-embedded cervical tissue samples. Among the 25 cervical cancers, 21 cases were squamous cancer and 4 were adenocarcinoma; 15 patients had a tumor stage of FIGO IA1 to IB1 and 10 had a tumor stage of FIGO IB2 to IIA; 7 tumors were well differentiated (G1), 8 were moderately differentiated (G2) and 10 were poorly differentiated cancer (G3); 9 patients had lymphovascular space invasion (LVSI); 4 patients had pelvic lymph node metastasis; the tumor sizes were greater than $2 \mathrm{~cm}$ in 9 cases.

The IHC assays revealed that both Ang-1 and Ang-2 were highly expressed on tumor cells compared to normal cervical epithelia. The positive expression rate of Ang-1 in cervical cancer epithelia was 60\% (15/25) and Ang-2 was 68\% (17/25), while normal cervical epithelia had no positive staining. For the expression of Ang-1 and Ang-2 in endothelial cells, there was no significant difference between cervical cancer tissue specimens and normal controls (Fig. 3A, B). 
MVD, as assessed by CD34 staining, was significantly increased in cervical cancer compared

245

246

247

248

249

250

251

252

253

254

255

256

257

258

259

260

261

262

263

264

265

266

267

268

269

with normal (Fig. 3C). The patients with positive Ang-2 in cervical epithelia had significantly

elevated sAng-2 concentration when compared to those with negative Ang-2 expression in cervical epithelia (80.89 (15.54-431.7) pg/ml vs $24.66(1.86-299.3) \mathrm{pg} / \mathrm{ml})(P=0.029$, Fig. 3D). In addition, the sAng-1 concentration did not correlate with MVD (r=0.256, $P=0.138$, Fig. $3 \mathrm{E})$; the sAng-2 concentration positively correlated to MVD (r=0.440, $P=0.008$, Fig. 3F); the sAng1/sAng-2 ratio was negatively correlated to MVD ( $r=-0.516, P=0.002$, Fig. $3 \mathrm{G})$.

\subsection{High sAng-1/sAng-2 ratio predicts poorer prognosis in cervical cancer patients}

The prognostic value of sAng-1, -2 and sAng-1/sAng-2 was evaluated in 66 cervical cancer patients with follow-up data. The patients were followed up to October 2016. The median follow-up time was 33.62 months (range, 9-48 months). Of the 66 patients, 17 suffered from recurrence and 8 died. According to the ROC analyses, we choose $131.00 \mathrm{pg} / \mathrm{ml}, 156.40 \mathrm{pg} / \mathrm{ml}$, and 0.26 as the cut-off values of sAng-1, sAng-2 and sAng-1/sAng-2, respectively, as these values were associated with a specificity of $95 \%$ for discriminating between invasive cervical cancer and normal. We found that there were no significant differences in the PFS and OS between the patients with high versus low sAng-1 or sAng-2 (Fig. 4A-D). However, the sAng1/sAng-2 ratio was significantly associated with PFS (Fig. 4E, P=0.046) and OS (Fig. 4F, $\mathrm{P}=0.040)$ in cervical cancer patients. In the low sAng-1/sAng-2 ratio group, 7 of 14 (50.0\%) patients developed recurrent disease and 4 (28.6\%) patients died. The estimated 3-year PFS and OS were $42.86 \%$ and $71.43 \%$, respectively. In the high sAng-1/sAng-2 ratio group, 10 of 52 (19.23\%) patients had recurrence and 4 (7.7\%) patients died, resulting in an estimated 3-year PFS and OS of $78.96 \%$ and $91.55 \%$, respectively.

\section{Discussion}

In the present study, we found that the concentration of sAng- 2 was significantly increased in 
270

271

272

273

274

275

276

277

278

279

280

281

282

283

284

285

286

287

288

289

290

291

292

293

294

295

296

patients with cervical cancer compared with normal controls and CIN patients and the sAng1/sAng-2 ratio was decreasing during the malignant transformation of cervical epithelia.

Moreover, the sAng-2 and the sAng-1/sAng-2 ratio exhibit encouraging accuracies for diagnosis of a CSCC. In patients with cervical cancer, low sAng-1/sAng-2 ratio was significantly associated with advanced stage, poor differentiation, LVSI, high MVD and poor survival. These findings suggest that the ratio of serum Angiopoietin-2 to Angiopoietin-1 is a valuable diagnostic and prognostic biomarker in patients with cervical cancer.

Tumor angiogenesis is the most fundamental trait for tumor growth. Tumor nodules cannot derive nutrients through diffusion when greater than 1-2 $\mathrm{mm}^{3}$ (Hanahan \& Weinberg 2011; Jain $\&$ Carmeliet 2012). Previous reports on various type of cancer, such as colorectal cancer, gastric carcinoma, and glioma, have shown that elevated Ang-2 in these tumor cells resulted in increased MVD and stimulated tumor angiogenesis (Augustin et al. 2009; Fagiani \& Christofori 2013; Shim et al. 2007). Moreover, in melanoma, tumor cells secreted sAng-2 and expressed Tie-2 suggesting that Ang-2 may act as an autocrine regulator of tumor cell migration and invasion (Helfrich et al. 2009). We found that Ang-2 was expressed in tumor cells of cervical cancer tissues. The sAng-2 concentration also positively correlated with Ang-2 expression in epithelia and MVD of cervical tissues, suggesting that the concentration of sAng-2 may reflect the activity of blood vessel formation and soluble Ang- 2 secreted by cervical cancer cells may be the main source of serum sAng-2 of cervical cancer. Given that the sAng-2 and the sAng1/sAng-2 ratio were significantly associated with MVD in cervical cancer (Fig. 3F-G), they might be used to evaluate the response of tumor to anti-angiogenesis therapy in real-time. In metastatic colorectal cancer, sAng-2 was a candidate biomarker for outcome of patients treated with bevacizumab-containing therapy (Goede et al. 2010). Very recently, a study indicated bevacizumab reduced mortality by $30 \%$ in patients with recurrent, persistent, or metastatic cervical cancer when combined with chemotherapy compared with those who receive chemotherapy alone (Penson et al. 2015). The utility of sAng-2 and sAng-1/sAng-2 ratio as 
297 biomarkers to predict outcome of bevacizumab-containing treatment in advanced cervical cancer 298 should be further validated.

We found that sAng-2 concentration gradually increased and the ratio of sAng-1/ sAng-2 gradually decreased with the severity of cervical lesion from control to CIN to CSCC. The sAng1/sAng-2 ratio was also decreased in CADC patients compared with normal group. In a previous study, Kopczynska et al. assessed plasma concentrations of Ang-1and Ang-2 in 34 patients with cervical cancer and 20 healthy volunteers. They reported that plasma concentrations of Ang-1 and Ang-2 were significantly higher in cervical cancer patients than in controls. Moreover, the plasma Ang-1/Ang-2 ratios was increased in cervical cancer compared with control and was higher in stage I than in stage II-III (Kopczynska et al. 2009). The increase in circulating Ang-2 in patients with cervical cancer was confirmed by our findings. However, the change in Ang1/Ang-2 ratio reported by Kopczynska et al. was opposite to that shown in our study and we did not find significant differences in Ang-1 concentration between control, CIN, and cervical cancer. These inconsistencies may be related to the differences in study population and the materials used in ELISA. In addition, the influence of anticoagulants used during blood collection on plasma angiopoietin detection cannot be excluded.

The ability of sAng-2 concentration and the ratio of sAng-1 to sAng-2 to distinguish between normal, CIN, and cervical cancer was similar to results reported in epithelial ovarian cancer, where the area under the curve for serum Ang-2 in ROC analysis was 0.75 to differentiate ovarian cancer from benign or borderline ovarian tumors (Sallinen et al. 2014; Sallinen et al. 2010). In melanoma, use of $90 \%$ quantile of healthy controls as a cutoff value could discriminate well between control individuals and individuals with melanoma stage III or IV patients (Helfrich et al. 2009). sAng-2 was elevated in patients with hepatocellular carcinoma, suggesting the potential use of angiopoietin-2 as a valuable diagnostic biomarker for the detection of hepatocellular carcinoma (Bouattour et al. 2015). Our findings suggest that sAng-2 concentration 
324

325

326

327

328

329

330

331

332

333

334

335

336

337

338

339

340

341

342

343

344

345

346

347

348

349

350

and the ratio of sAng-1 to sAng-2 may be novel diagnostic biomarkers for the detection of normal, CIN and cervical cancer. Additional research to determine cutoff values based on population characteristics are being explored.

Both sAng-1 and sAng-2 have been found to be involved in tumor growth and progression. In most conditions, elevated sAng-1 played anti-tumorigenic effects, whereas upregulated sAng-2 stimulated tumor growth and progression (Augustin et al. 2009; Eklund \& Saharinen 2013; Fagiani \& Christofori 2013; Shim et al. 2007). In some tumors, such as glioblastoma, breast cancer, hepatocellular carcinoma and pancreatic cancer, both sAng-1 and sAng-2 are upregulated (Augustin et al. 2009; Fagiani \& Christofori 2013; Shim et al. 2007; Eklund \& Saharinen 2013). Elevated sAng-1 was found in lung cancer and epithelial ovarian cancer, yet no relation was found with diagnosis and prognosis of tumor (Park et al. 2009; Sallinen et al. 2014). On the contrary, patient with papillary thyroid cancer were found to have significantly decreased sAng-1 compared with the normal control (Makki et al. 2013). We found that sAng-2, but not sAng-1 was increased, suggesting that factors driving tumor angiogenesis may be unique to tumor types.

Elevated sAng-2 tightly correlated with lymphatic and liver metastasis and shorter survival in pancreatic ductal adenocarcinoma (Schulz et al. 2011) and neuroendocrine tumor patients (Detjen et al. 2010; Helfrich et al. 2009). sAng-2 has also been suggested to be a useful biomarker in melanoma as progression and metastasis correlated with tumor load and overall survival (Helfrich et al. 2009). In epithelial ovarian cancer, the serum concentration of Ang-2 predicted poor overall survival (Sallinen et al. 2014; Sallinen et al. 2010). In our study, we found that lower sAng-1 in patients with cervical cancer correlated with more advanced cervical cancer, including higher stage, larger tumor size and LVSI. We also found higher sAng-2 levels in patients with poorly differentiated cervical cancer. Overall, the ratio of sAng-1/sAng-2 was predictive of PFS and OS, showing that patient with cervical cancer and a lower sAng-1/sAng-2 had a poorer prognosis. 
352 The present study assessed for the first time the serum concentrations of angiopoietins and sAng-

353 1/sAng-2 ratio in healthy women and patients with CIN and invasive cervical cancer and

354

355

356

357

358

359

360

361

362

363

364

365

366

367

368

369

370

371

372

373

374

375

376

377

378

379

380

381

382

383

evaluated the diagnostic and prognostic power of sAng-1, sAng-2 and sAng-1/sAng-2 in cervical cancer. A major limitation of this study is the small sample size of cervical cancer $(N=77)$, particularly that of CADC $(\mathrm{N}=16)$, which may be associated with selection bias and low statistical power. In addition, the follow-up periods of the patients were relatively short. Multicenter studies with long-term follow-up are needed to verify our findings.

\section{Conclusion}

In summary, sAng-2 was significantly increased in cervical cancer patients and a low sAng1/sAng-2 ratio was highly effective of discriminating cervical cancer patients from normal control and predictive of poor survival in patients with cervical cancer. These findings suggest that the sAng-1/sAng-2 ratio is a potential diagnostic and prognostic biomarker for cervical cancer. Further studies to validate these findings and explore the utility of anti-angiogenesis therapeutic targets for treatment of advanced or recurrent cervical cancer are needed.

\section{References:}

Augustin HG, Koh GY, Thurston G, and Alitalo K. 2009. Control of vascular morphogenesis and homeostasis through the angiopoietin-Tie system. Nat Rev Mol Cell Biol 10:165-177. 10.1038/nrm2639

Bouattour M, Payance A, and Wassermann J. 2015. Evaluation of antiangiogenic efficacy in advanced hepatocellular carcinoma: Biomarkers and functional imaging. World J Hepatol 7:2245-2263. 10.4254/wjh.v7.i20.2245

Detjen KM, Rieke S, Deters A, Schulz P, Rexin A, Vollmer S, Hauff P, Wiedenmann B, Pavel M, and Scholz A. 2010. Angiopoietin-2 promotes disease progression of neuroendocrine tumors. Clin Cancer Res 16:420-429. 10.1158/1078-0432.ccr-09-1924

Ebos JM, and Kerbel RS. 2011. Antiangiogenic therapy: impact on invasion, disease progression, and metastasis. Nat Rev Clin Oncol 8:210-221. 10.1038/nrclinonc.2011.21

Eklund L, and Saharinen P. 2013. Angiopoietin signaling in the vasculature. Exp Cell Res 319:1271-1280. 10.1016/j.yexcr.2013.03.011

Fagiani E, and Christofori G. 2013. Angiopoietins in angiogenesis. Cancer Lett 328:18-26. 10.1016/j.canlet.2012.08.018

Ferlay J, Soerjomataram I, Dikshit R, Eser S, Mathers C, Rebelo M, Parkin DM, Forman D, and Bray F. 2015. 
Cancer incidence and mortality worldwide: sources, methods and major patterns in GLOBOCAN 2012. Int J Cancer 136:E359-386. 10.1002/ijc.29210

Gerald D, Chintharlapalli S, Augustin HG, and Benjamin LE. 2013. Angiopoietin-2: an attractive target for improved antiangiogenic tumor therapy. Cancer Res 73:1649-1657. 10.1158/0008-5472.can-12-4697

Goede V, Coutelle O, Neuneier J, Reinacher-Schick A, Schnell R, Koslowsky TC, Weihrauch MR, Cremer B, Kashkar H, Odenthal M, Augustin HG, Schmiegel W, Hallek M, and Hacker UT. 2010. Identification of serum angiopoietin-2 as a biomarker for clinical outcome of colorectal cancer patients treated with bevacizumab-containing therapy. Br J Cancer 103:1407-1414. 10.1038/sj.bjc.6605925

Hanahan D, and Weinberg RA. 2011. Hallmarks of cancer: the next generation. Cell 144:646-674. 10.1016/j.cell.2011.02.013

Helfrich I, Edler L, Sucker A, Thomas M, Christian S, Schadendorf D, and Augustin HG. 2009. Angiopoietin-2 levels are associated with disease progression in metastatic malignant melanoma. Clin Cancer Res 15:1384-1392. 10.1158/1078-0432.ccr-08-1615

Jain RK, and Carmeliet P. 2012. SnapShot: Tumor angiogenesis. Cell 149:1408-1408 e1401. 10.1016/j.cell.2012.05.025

Kerbel RS. 2015. A Decade of Experience in Developing Preclinical Models of Advanced- or Early-Stage Spontaneous Metastasis to Study Antiangiogenic Drugs, Metronomic Chemotherapy, and the Tumor Microenvironment. Cancer J 21:274-283. 10.1097/ppo.0000000000000134

Kopczynska E, Makarewicz R, Biedka M, Kaczmarczyk A, Kardymowicz H, and Tyrakowski T. 2009. Plasma concentration of angiopoietin-1, angiopoietin-2 and Tie-2 in cervical cancer. Eur J Gynaecol Oncol 30:646649 .

Makki FM, Taylor SM, Shahnavaz A, Leslie A, Gallant J, Douglas S, Teh E, Trites J, Bullock M, Inglis K, Pinto DM, and Hart RD. 2013. Serum biomarkers of papillary thyroid cancer. J Otolaryngol Head Neck Surg 42:16. 10.1186/1916-0216-42-16

Park JH, Choi H, Kim YB, Kim YS, Sheen SS, Choi JH, Lee HL, Lee KS, Chung WY, Lee S, Park KJ, Hwang SC, Lee KB, and Park KJ. 2009. Serum angiopoietin-1 as a prognostic marker in resected early stage lung cancer. Lung Cancer 66:359-364. 10.1016/j.lungcan.2009.03.002

Penson RT, Huang HQ, Wenzel LB, Monk BJ, Stockman S, Long HJ, 3rd, Ramondetta LM, Landrum LM, Oaknin A, Reid TJ, Leitao MM, Method M, Michael H, and Tewari KS. 2015. Bevacizumab for advanced cervical cancer: patient-reported outcomes of a randomised, phase 3 trial (NRG Oncology-Gynecologic Oncology Group protocol 240). Lancet Oncol 16:301-311. 10.1016/s1470-2045(15)70004-5

Sallinen H, Heikura T, Koponen J, Kosma VM, Heinonen S, Yla-Herttuala S, and Anttila M. 2014. Serum angiopoietin-2 and soluble VEGFR-2 levels predict malignancy of ovarian neoplasm and poor prognosis in epithelial ovarian cancer. BMC Cancer 14:696. 10.1186/1471-2407-14-696

Sallinen H, Heikura T, Laidinen S, Kosma VM, Heinonen S, Yla-Herttuala S, and Anttila M. 2010. Preoperative angiopoietin-2 serum levels: a marker of malignant potential in ovarian neoplasms and poor prognosis in epithelial ovarian cancer. Int J Gynecol Cancer 20:1498-1505. 10.1111/IGC.0b013e3181f936e3

Scholz A, Lang V, Henschler R, Czabanka M, Vajkoczy P, Chavakis E, Drynski J, Harter PN, Mittelbronn M, Dumont DJ, Plate KH, and Reiss Y. 2011. Angiopoietin-2 promotes myeloid cell infiltration in a beta(2)integrin-dependent manner. Blood 118:5050-5059. 10.1182/blood-2011-03-343293

Schulz P, Fischer C, Detjen KM, Rieke S, Hilfenhaus G, von Marschall Z, Bohmig M, Koch I, Kehrberger J, Hauff 
425

426

427

428

429

430

431

432

433

434

435

436

437
P, Thierauch KH, Alves F, Wiedenmann B, and Scholz A. 2011. Angiopoietin-2 drives lymphatic metastasis of pancreatic cancer. FASEB J 25:3325-3335. 10.1096/fj.11-182287

Shim WS, Ho IA, and Wong PE. 2007. Angiopoietin: a TIE(d) balance in tumor angiogenesis. Mol Cancer Res 5:655-665. 10.1158/1541-7786.mcr-07-0072

Staton CA, Hoh L, Baldwin A, Shaw L, Globe J, Cross SS, Reed MW, and Brown NJ. 2011. Angiopoietins 1 and 2 and Tie-2 receptor expression in human ductal breast disease. Histopathology 59:256-263. 10.1111/j.13652559.2011.03920.x

Yang P, Chen N, Jia JH, Gao XJ, Li SH, Cai J, and Wang Z. 2015. Tie-1: A potential target for anti-angiogenesis therapy. J Huazhong Univ Sci Technolog Med Sci 35:615-622. 10.1007/s11596-015-1479-1

Yang S, Cheng H, Cai J, Cai L, Zhang J, and Wang Z. 2009. PlGF expression in pre-invasive and invasive lesions of uterine cervix is associated with angiogenesis and lymphangiogenesis. APMIS 117:831-838.

$10.1111 / \mathrm{j} .1600-0463.2009 .02538 . \mathrm{x}$ 
Figure 1

sAng-2 concentration and sAng-1/sAng-2 ratio are altered in patients with cervical neoplasia

sAng- 1 and sAng- 2 concentrations in 43 patients of normal control, 44 CIN patients, and 77 cervical cancer patients (62 CSCC patients and 15 CADC patients) were determined by ELISA. The differences in Ang-1 (A), sAng-2 (B), and the sAng-1/sAng-2 ratio (C) between groups are shown.
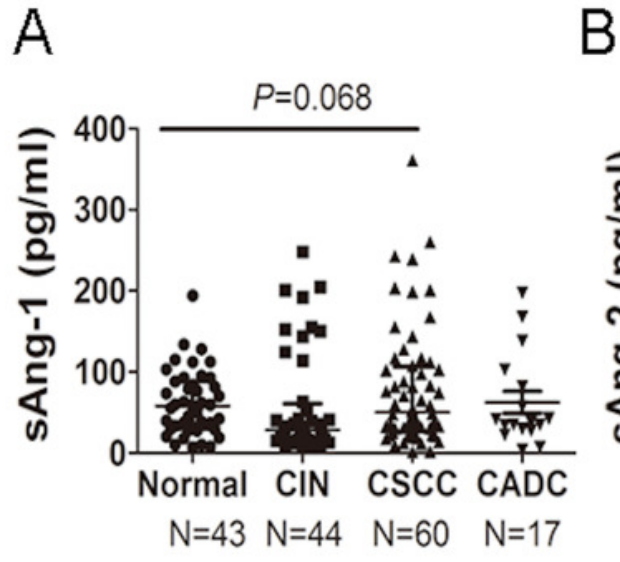

B

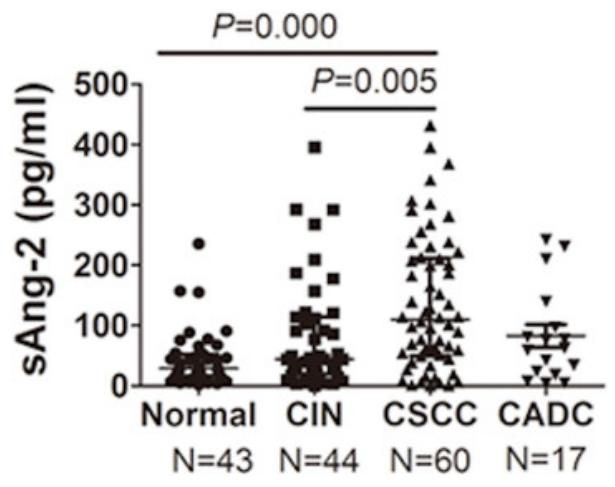

C

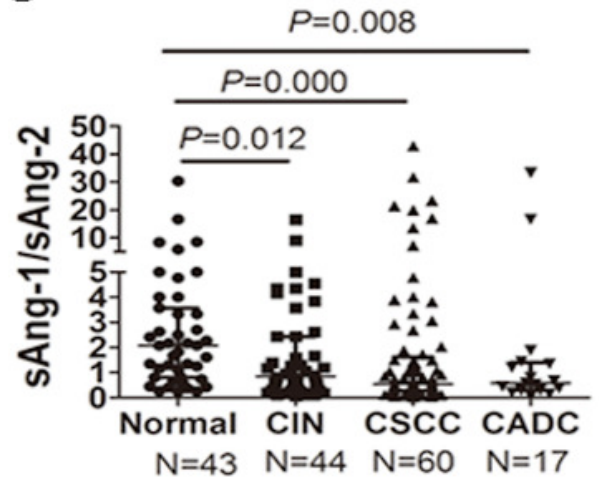


Figure 2

sAng- 2 concentration and the sAng-1/sAng-2 ratio are valuable diagnostic biomarkers for cervical lesions

The ROC curves of sAng-2 for discriminating CSCC from normal (A), CSCC from CIN (B), CSCC from normal and CIN (C), CIN and CSCC from normal (D), and invasive cervical cancer from normal (E). The cutoff values of sAng-2 and corresponding sensitivity and specificity to distinguish CSCC from normal are shown (F). The ROC curves of sAng-1/ sAng-2 for discriminating CIN from normal (G), CSCC from normal (H), CIN and CSCC from normal (I) and invasive cervical cancer from normal $(\boldsymbol{J})$. The cutoff values of sAng-1/ sAng-2 and corresponding sensitivity and specificity to distinguish CSCC from normal are listed (K).

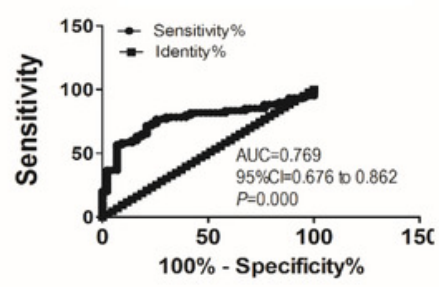

$E$

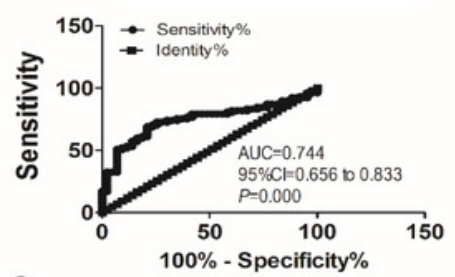

G

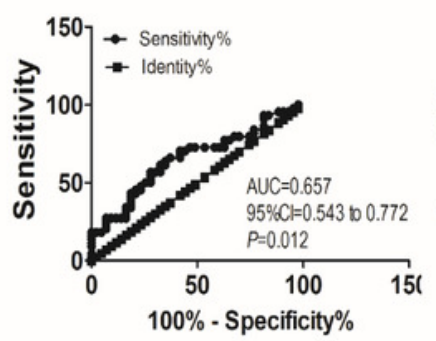

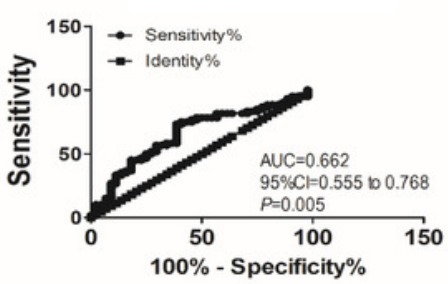

$\mathrm{F}$

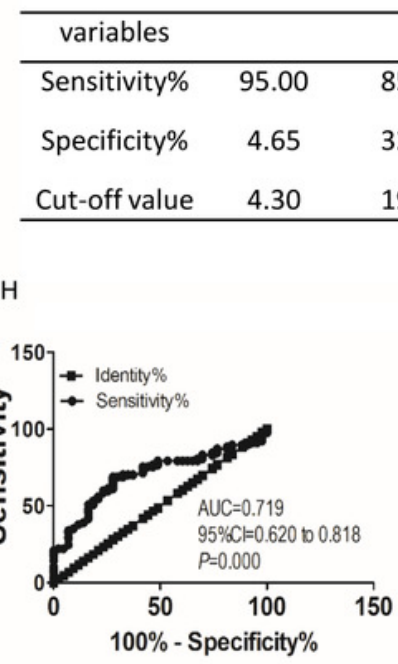

C

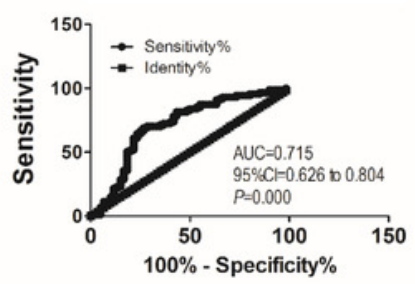

D

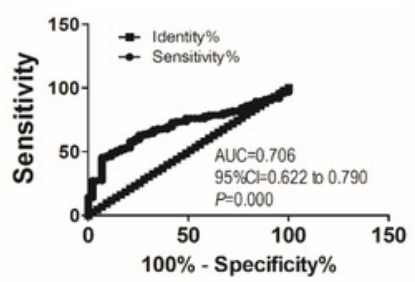
$\mathrm{K}$

\begin{tabular}{cccccccc}
\hline variables & & \multicolumn{7}{c}{ sAng-1/sAng-2 for normal to CSCC } \\
\hline Sensitivity\% & 22.08 & 35.06 & 50.65 & $\mathbf{6 8 . 8 3}$ & 75.32 & 85.71 & 94.81 \\
& & & & & & & \\
Specificity\% & 95.36 & 90.70 & 81.40 & $\mathbf{7 2 . 0 9}$ & 53.49 & 23.26 & 2.32 \\
& & & & & & & \\
Cut-off value & 0.26 & 0.39 & 0.58 & 1.09 & 1.70 & 3.80 & 20.70 \\
\hline
\end{tabular}




\section{Figure 3}

sAng-2 concentration positively relates with Ang-2 expression on the epithelia and MVD in cervical tissues

Representative immunohistochemical staining of Ang-1(A), Ang-2 (B) and CD34 (C) in 25 cervical cancer tissue specimens and 10 normal controls. Black arrows denote positively stained epithelial cells, whereas red arrows denote positively staining endothelial cells, all appearing brown. Scale bar, $20 \mu \mathrm{m}$. (D) sAng-2 is significantly higher in the patients with positive Ang-2 expression on cervix epithelia than those with negative Ang-2 expression. The scatter diagrams show the correlations of sAng-1 (E), sAng-2 (F) and sAng-1/ sAng-2 ratio (G) to MVD in the 35 cervical tissue specimens.

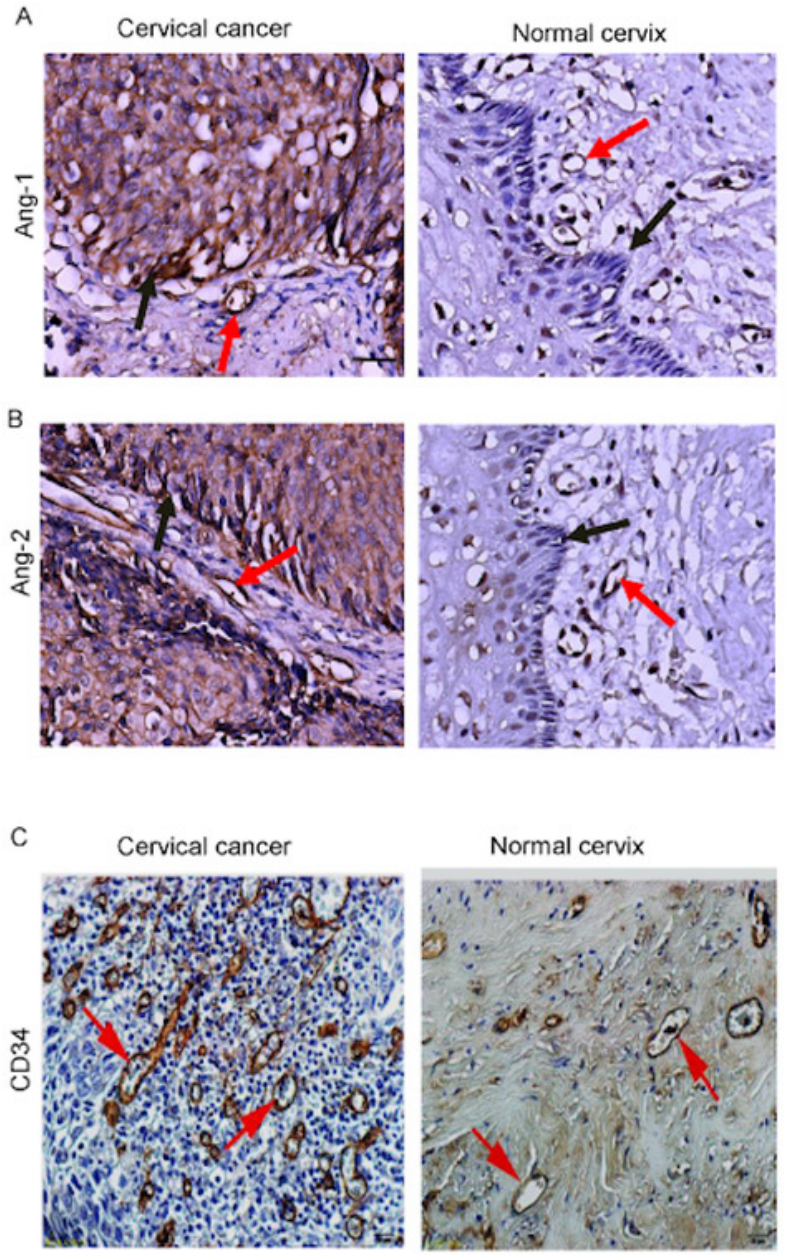

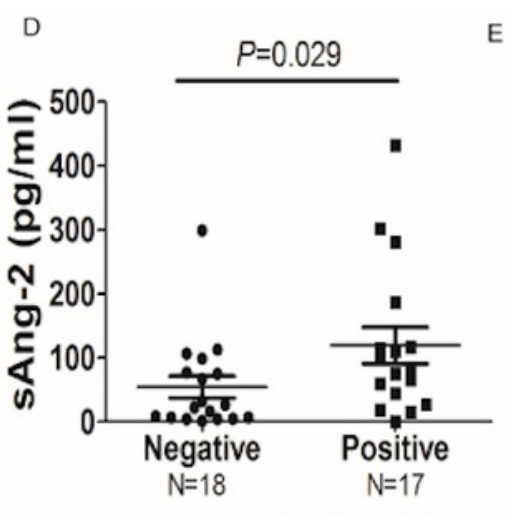

Expression of Ang-2 on epithelia

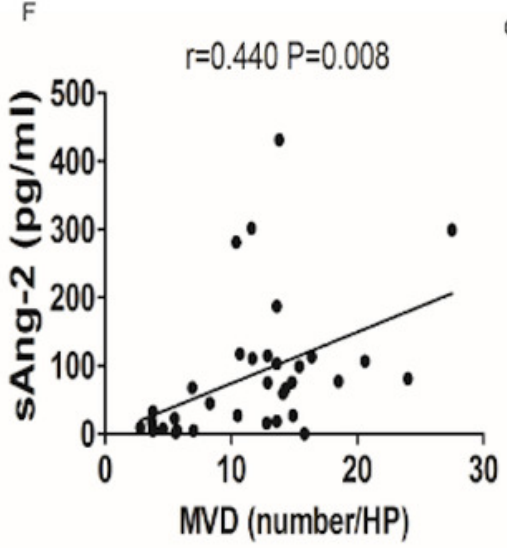

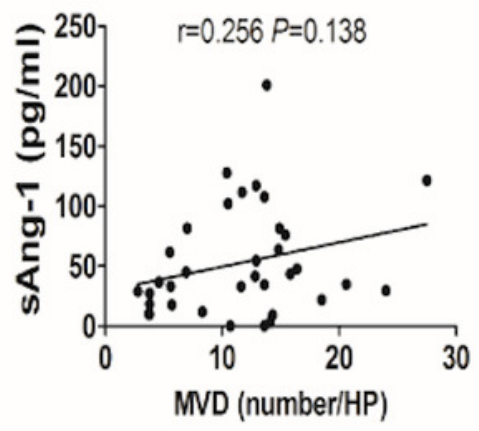

G

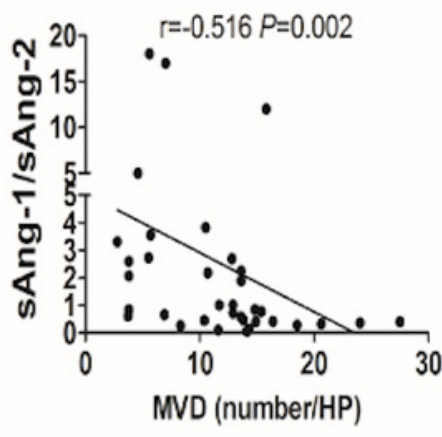




\section{Figure 4}

High sAng-1/sAng-2 ratio predicts poorer survival in cervical cancer patients

Kaplan-Meier survival analysis of the progression-free survival and the overall survival among 66 cervical cancer patients stratified by sAng-1(A, B), sAng-2 (C, D) and sAng-1/sAng-2 ratio (E, F).

A

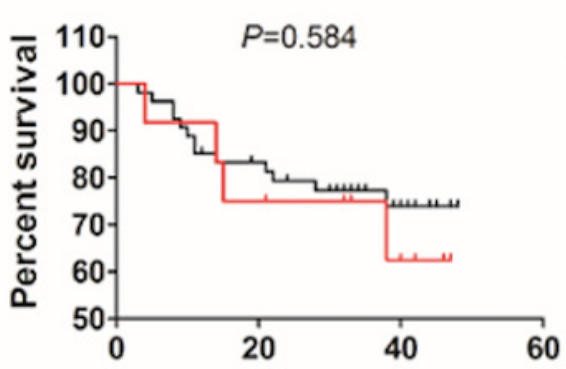

Progression free survival (months)

C

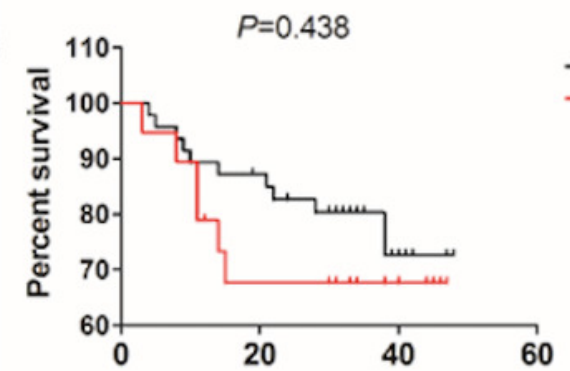

Progression free survival (months)

$E$

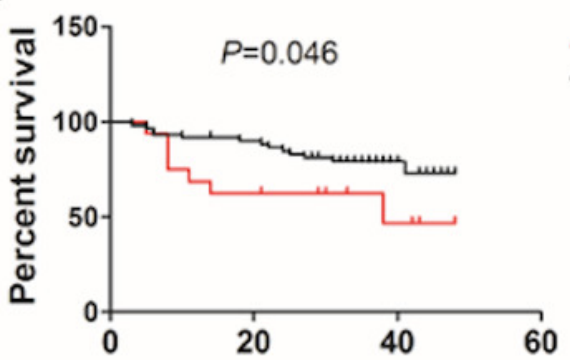

Progression free survival (months)
B

- High sang-1

- Low SAng-1

- Low sAng-2

- High SAng-2

D

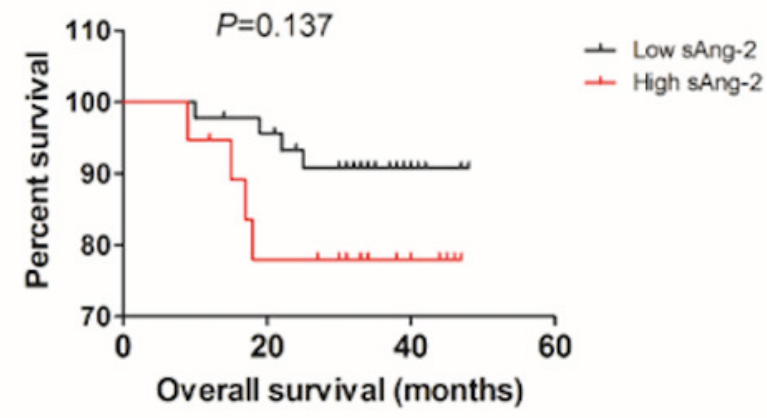

$\mathrm{F}$

- Low sAng- $1 /$ sAng-2

- High s.Ang-1/sAng-2

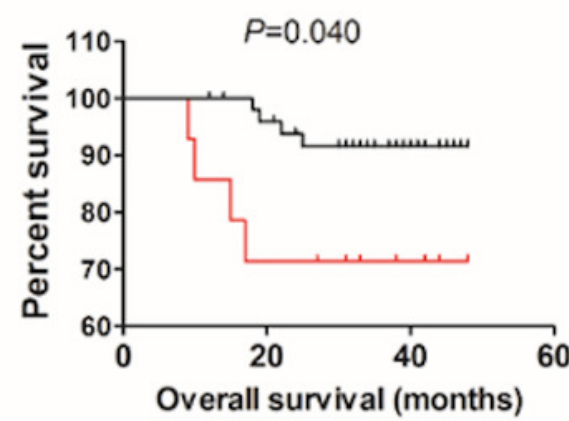

- High SAng-1

- Low SAng-1
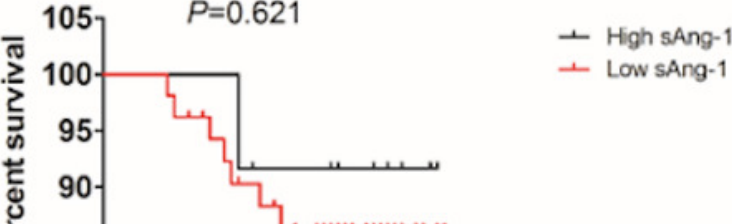

85

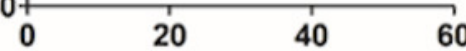

Overall survival (months)

- High SAng- $1 /$ sAng- 2
Overall survival (months) 


\section{Table $\mathbf{1}$ (on next page)}

The characteristics and serum angiopoietins in patients with cervical canc er $(N=77)$. 
1 Table 1 The characteristics and serum angiopoietins in patients with cervical cancer $(N=77)$.

\begin{tabular}{|c|c|}
\hline Parameters & Cases $(\%)$ \\
\hline \multicolumn{2}{|l|}{ Age (year) } \\
\hline Median (range) & $46(28-68)$ \\
\hline$\leq 45$ & $37(48.1)$ \\
\hline$>45$ & $40(51.9)$ \\
\hline \multicolumn{2}{|l|}{ FIGO stage } \\
\hline IA1-IA2 & $3(3.9)$ \\
\hline IB1 & $38(49.4)$ \\
\hline IB2-IIA2 & $20(25.9)$ \\
\hline IIB & $12(15.6)$ \\
\hline IIIA- IV & $4(5.20)$ \\
\hline \multicolumn{2}{|l|}{ Pathological type } \\
\hline Squamous carcinoma & $61(79.2)$ \\
\hline Adenocarcinoma & $16(20.8)$ \\
\hline \multicolumn{2}{|l|}{ Differentiation } \\
\hline Well differentiated & $19(24.7)$ \\
\hline Moderately differentiated & $31(40.2)$ \\
\hline Poorly differentiated & $27(35.1)$ \\
\hline \multicolumn{2}{|l|}{ Pelvic lymph node metastasis } \\
\hline Negative & $40(51.9)$ \\
\hline Positive & $21(27.3)$ \\
\hline Unknown & $16(20.8)$ \\
\hline \multicolumn{2}{|l|}{ Tumor size } \\
\hline$\leq 2 \mathrm{~cm}$ & $52(67.5)$ \\
\hline$>2 \mathrm{~cm}$ & $25(32.5)$ \\
\hline \multicolumn{2}{|c|}{ Lymphovascular space invasion } \\
\hline Negative & $48(62.3)$ \\
\hline Positive & $29(37.7)$ \\
\hline \multicolumn{2}{|l|}{ sAng-1 (pg/ml) } \\
\hline Median (range) & $47.81(1.57-361.21)$ \\
\hline High & $14(18.2)$ \\
\hline Low & $63(81.8)$ \\
\hline \multicolumn{2}{|l|}{ sAng-2 (pg/ml) } \\
\hline Median (range) & $89.94(1.09-431.68)$ \\
\hline High & $24(31.2)$ \\
\hline Low & $53(68.8)$ \\
\hline \multicolumn{2}{|l|}{ sAng-1/sAng-2 } \\
\hline Median (range) & $0.54(0.02-42.91)$ \\
\hline High & $60(77.9)$ \\
\hline
\end{tabular}




$2 \longdiv { \text { Low } } 1 7 ( 2 2 . 1 )$




\section{Table 2 (on next page)}

The correlation between sAng-1, sAng-2, sAng-1/sAng-2 ratio and clinicopathological characteristics in cervical cancer $(N=77)$. 
1 Table 2 The correlation between sAng-1, sAng-2, sAng-1/sAng-2 ratio and clinicopathological characteristics in cervical cancer

\begin{tabular}{|c|c|c|c|c|c|c|c|}
\hline \multirow{2}{*}{ Variable } & \multirow{2}{*}{ Cases } & sAng-1 (pg/ml) & \multirow{2}{*}{$P$} & sAng-2 (pg/ml) & \multirow{2}{*}{$P$} & sAng-1/sAng-2 & \multirow{2}{*}{$P$} \\
\hline & & Median (range) & & Median (range) & & Median (range) & \\
\hline \multicolumn{8}{|l|}{ Age (year) } \\
\hline$\leq 45$ & 37 & $59.92(1.57-361.21)$ & 0.266 & $66.28(1.09-431.68)$ & 0.203 & $0.77(0.02-33.33)$ & 0.101 \\
\hline$>45$ & 40 & $37.96(11.84-253.59)$ & & $113.70(1.68-368.08)$ & & $0.46(0.08-50.00)$ & \\
\hline \multicolumn{8}{|l|}{ FIGO stage } \\
\hline IA1-IB1 & 41 & $59.92(1.57-361.21)$ & 0.046 & $71.38(1.19-431.68)$ & 0.691 & $0.85(0.02-23.35)$ & 0.010 \\
\hline$\geq \mathrm{IB} 2$ & 36 & $36.80(1.91-253.59)$ & & $94.01(1.09-306.77)$ & & $0.37(0.04-42.91)$ & \\
\hline \multicolumn{8}{|l|}{ Pathological type } \\
\hline Squamous carcinoma & 61 & $48.86(1.57-361.21)$ & 0.920 & $101.99(1.09-431.68)$ & 0.085 & $0.53(0.02-50.00)$ & 0.444 \\
\hline Adenocarcinoma & 16 & $39.63(3.68-197.66)$ & & $51.92 \quad(4.37-242.26)$ & & $0.69(0.06-33.33)$ & \\
\hline Differentiation & & & & & & & \\
\hline
\end{tabular}

$2(N=77)$. 


\begin{tabular}{|c|c|c|c|c|c|c|c|c|}
\hline Well & moderately & 50 & $51.90(3.68-361.21)$ & 0.364 & $64.31(1.09-431.68)$ & 0.002 & $0.88(0.06-31.71)$ & 0.000 \\
\hline \multicolumn{9}{|l|}{ differentiated } \\
\hline Poorly differentiated & & 27 & $42.47(1.57-260.11)$ & & $152.49(5.91-368.08)$ & & $0.27(0.02-42.91)$ & \\
\hline \multicolumn{9}{|c|}{ Pelvic lymph node metastasis } \\
\hline Negative & & 40 & $81.68(9.52-253.59)$ & 0.421 & $65.70(1.09-431.68)$ & 0.476 & $0.78(0.14-42.91)$ & 0.281 \\
\hline Positive & & 21 & $45.14(3.68-361.21)$ & & $83.67(1.68-395.47)$ & & $0.77(0.02-23.35)$ & \\
\hline \multicolumn{9}{|l|}{ Tumor size } \\
\hline$\leq 2 \mathrm{~cm}$ & & 52 & $59.92(1.57-361.21)$ & 0.034 & $101.99(1.19-431.68)$ & 0.306 & $0.58(0.02-42.91)$ & 0.286 \\
\hline$>2 \mathrm{~cm}$ & & 25 & $34.41(1.91-203.59)$ & & $69.23(1.09-291.58)$ & & $0.35(0.04-31.71)$ & \\
\hline \multicolumn{9}{|c|}{ Lymphovascular space invasion } \\
\hline Negative & & 48 & $56.12(1.57-361.21)$ & 0.039 & $80.66(1.19-431.68)$ & 0.328 & $0.71(0.02-50.00)$ & 0.013 \\
\hline Positive & & 29 & $38.12(1.91-121.64)$ & & $114.26(1.09-299.27)$ & & $0.41(0.04-20.00)$ & \\
\hline
\end{tabular}

